\title{
Factors involved in the production of amblyopia
}

\author{
G. K. VON NOORDEN \\ From the Department of Ophthalmology, Baylor College of Medicine, Houston, Texas, U.S.A.
}

If amblyopia is defined as reduced visual acuity in an eye which appears normal on examination, then it is necessary to distinguish between several related, similar, or different conditions that fit this definition. Six years ago I proposed a classification of amblyopia in which the various factors leading to its development were enumerated in the light of our clinical knowledge at that time (von Noorden, 1967). Meanwhile, with the advent of modern neurophysiological techniques, amblyopia has become a favourite research subject of neurophysiologists and neuroanatomists, and the effect of abnormal visual experience early in life on visual functions of the adult organism is currently being explored on a large scale in lower animals and in monkeys. Some of this information has direct bearing on our concept of amblyopia as it occurs in human patients.

The purpose of this paper is to discuss the extent to which our conventional concept of the various factors that cause amblyopia in humans may have to be modified in light of this new information. This discussion will be limited to amblyopia in association with strabismus, anisometropia, and form vision deprivation; organic amblyopia and that associated with nystagmus, achromatopsia, or nutritional deficiencies will not be considered.

Let us briefly review the factors that have been held responsible for the production of each type of amblyopia.

\section{Strabismic amblyopia}

The classical view is that strabismic amblyopia develops as an adaptive mechanism against visual discomfort caused by diplopia and image confusion when the eyes are misaligned. The ocular deviation causes dissimilar images to be presented to the two foveae; and since the two foveae have a common visual direction, the patient will see these objects superimposed (Fig. I, opposite). The perceptual conflict arising from this situation has been aptly referred to as "confusion" and in principle is no different from retinal rivalry in normal individuals when dissimilar images are presented in a haploscopic arrangement to each eye. Thus, as commonly assumed, it is not diplopia but rather the dissimilarity of foveal images in the two eyes that causes amblyopia in a strabismic patient.

\section{Anisometropic amblyopia}

In anisometropic amblyopia the images on the two foveae originate from the same visual object (Fig. 2). They are of different sharpness, however, or, if the refractive error is corrected, of different size (aniseikonia). As in strabismic amblyopia, the dissimilarity of foveal images is incompatible with foveal fusion, and the image from one eye, usually the more ametropic eye, is suppressed. From these observations, it is apparent that the mech- 


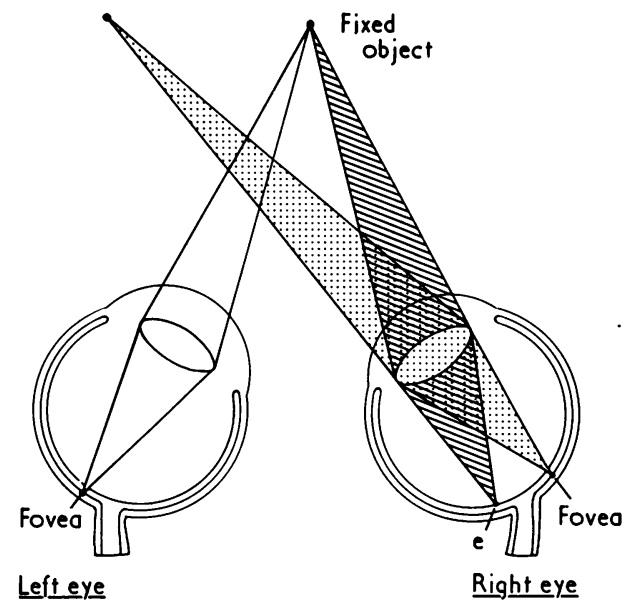

FIG. I

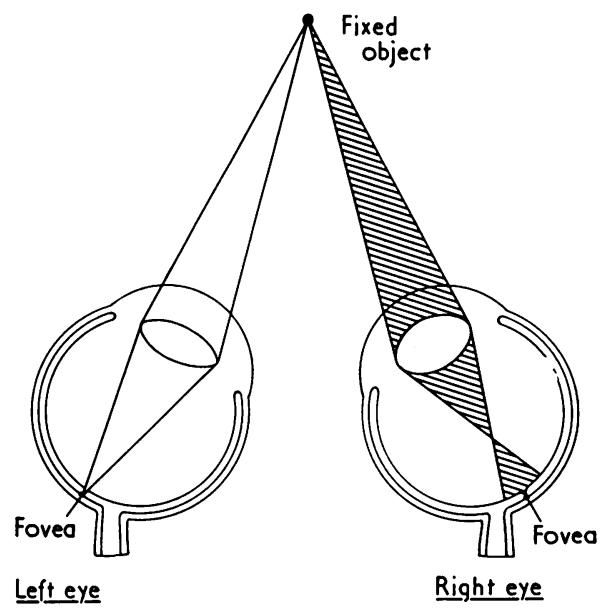

FIG. 2

FIG. I Strabismic amblyopia. The image of the visual object fixed by the left eye falls on nasal retinal elements (e) of the right esotropic eye (diplopia). The image of a second object, located to the left of the object fixed by the left eye, falls on the fovea of the right eye (confusion)

FIG. 2 Anisometropic amblyopia. The fovea of the right myopic eye receives a blurred image of the object fixed by the left emmetropic eye

anism leading to amblyopia in patients with strabismus and anisometropia is not as different in principle as it may seem on first glance.

\section{Form vision deprivation amblyopia}

This form of amblyopia may occur in one or both eyes as a result of deprivation of normal visual experience early in life by corneal opacities, congenital cataracts, ptosis, or other obstacles to normal retinal stimulation (von Noorden and Maumenee, 1968) (Fig. 3).

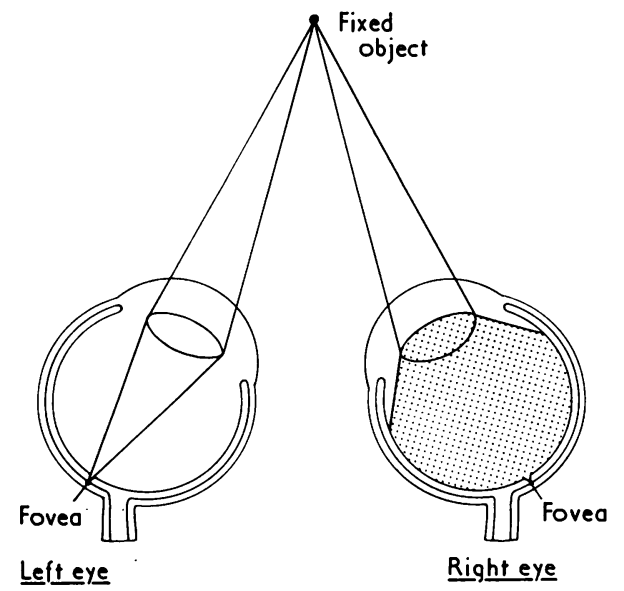

FIG. 3 Form vision deprivation amblyopia. The cataract in the right eye prevents image formation on the fovea; only diffuse light can enter the eye

Interestingly, the amblyopia is more severe after unilateral than after bilateral visual deprivation. The clinician has always associated this form of amblyopia with disuse of the eye, and we have suggested that the term "amblyopia ex anopsia", formerly used to describe all forms of amblyopia, should be reserved to describe this entity. 
At first glance, fundamental differences appear to exist in the aetiology of strabismic and anisometropic amblyopia on the one hand and amblyopia ex anopsia on the other. In the first instance, there is no question that the deviated or more ametropic eye participates, at least to some degree, in visual activity. Images are formed on the fovea and the retinal periphery; and except for extreme forms of strabismus, the amount of light entering the eye is the same as that which stimulates the fixing eye. Both conditions are reversible if appropriate treatment is instituted at a young age. Conversely, in patients with amblyopia ex anopsia, one or both eyes have been prevented from receiving adequate stimulation during the age of visual immaturity. Amblyopia ex anopsia, except for transient forms (occlusion amblyopia), does not usually respond to therapy, and the visual defect as a rule is more severe than in patients with strabismus or anisometropia.

In view of these aetiological differences and variations in clinical manifestations and severity, we must ask whether the basic mechanisms underlying these conditions also are different. Teleologically, this appears unlikely, and we shall now explore the possible existence of aetiological denominators common to strabismic, anisometropic, and form vision deprivation amblyopia.

First, it is significant that none of these conditions develops in the adult patient but that they occur at an age at which the integrity of the visual system is susceptible to modification of visual experience. Although this time period varies in individual patients, clinical experience indicates that it ranges from birth to approximately $4 \frac{1}{2}$ to 5 years of age for all forms of amblyopia (von Noorden, i 973a).

Secondly, a certain degree of visual deprivation occurs, in fact, with all three forms of amblyopia. Ikeda and Wright ( I972a) studied the effect of defocusing a spot stimulus on the gradient of excitation across the receptive field of ganglion cells in the cat retina and found that central retinal ganglion cells require a sharply focused image in order to respond. Since accommodation in strabismus is determined by the requirements of the fixing eye, the image falling on the fovea of the deviating eye is not in focus (Figs $4 \mathrm{~A}$ and $\mathrm{B}$ ) except
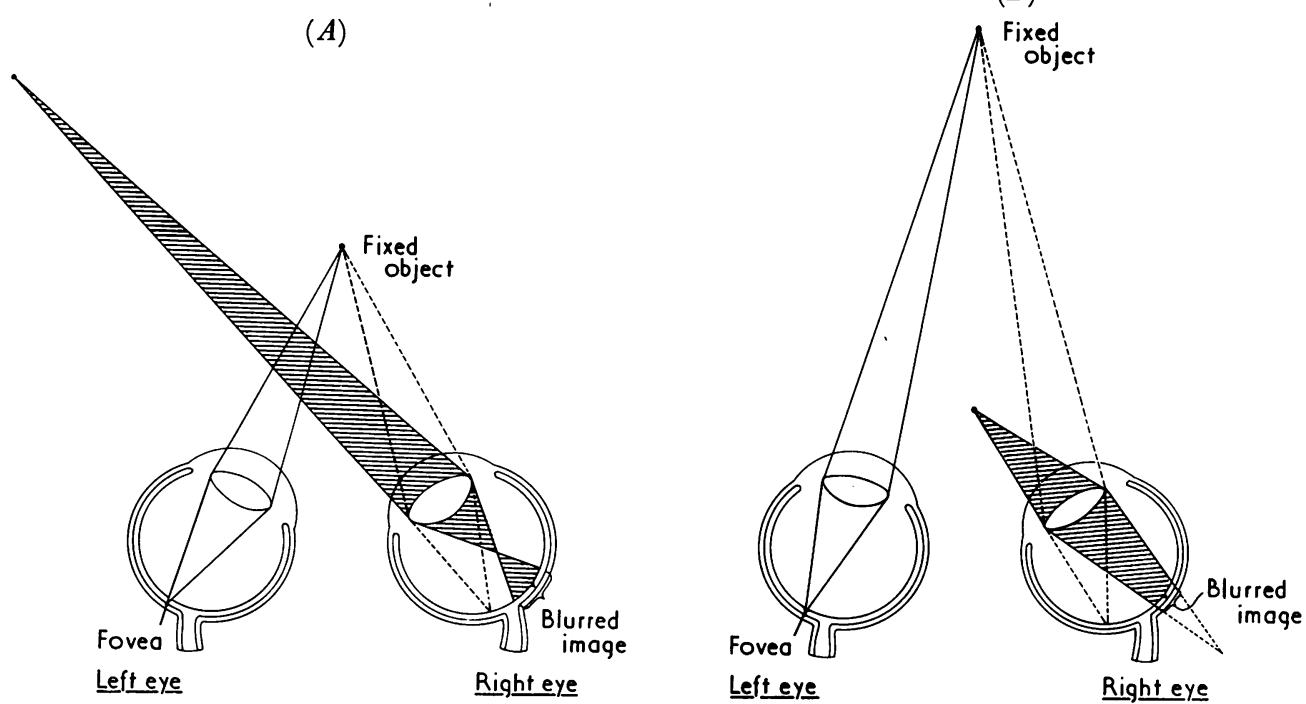

FIG. 4 Form vision deprivation in strabismus. Accommodative requirements of the deviating right eye are determined by the fixing left eye. The fovea of the deviating eye will receive a blurred image of objects located further distant $(A)$ or nearer $(B)$ than the fixed object. 
when visual objects in the line of sight of either eye are located exactly at the same distance from the patient (Fig. I). Ikeda and Wright (I972a) pointed out that visual deprivation in strabismus may occur as a result of the habitual exposure of the fovea of the deviating eye to defocused images with low contrast and detail.

Further evidence that visual deprivation may be instrumental in producing human amblyopia other than that classically associated with lid closure or congenital cataract was provided by Mitchell, Freeman, Millodot, and Haegerstrom (I973), according to whom, subjects with ocular astigmatism habitually experience contours in one orientation clearer than in others, so that the visual system is deprived of sharp images of contours of certain orientations. Mitchell and others (1973) have established that, after full correction of the refractive error in astigmatic patients, large differences occur in acuity for gratings oriented in the vertical and horizontal meridian and that this defect is neural rather than optical in nature. The severity of this "meridional" amblyopia correlated well with the degree and orientation of the astimatism. This finding is of special significance in the light of the experiments of Hirsch and Spinelli (1970, 1971) and Blakemore and Cooper (1970), who have shown that the distribution of receptive field orientation can be modified by selective visual exposure during visual immaturity. It is reasonable to assume that the neural organization of the human visual cortex may be altered in a similar manner by uncorrected astigmatism. From these experiments, therefore, it appears that visual deprivation is not necessarily limited to amblyopia ex anopsia but also may play an active and possibly critical role in the production of strabismic and anisometropic amblyopia as well as causing meridional amblyopia in astigmatism.

However, the neurophysiological experiments of Wiesel and Hubel (1965a) and the anatomical studies of Guillery and Stelzner (1970) suggest that the functional integrity of the visual system may depend not only on the adequacy of afferent impulse activity but also on the interrelationship and possible interaction of the input received by each eye. In the light of these data, we must consider the possibility that abnormal binocular interaction, which has been shown to cause severe neurophysiological and anatomical anomalies of the visual system in animals, may be yet another cause of amblyopia. For instance, in strabismic patients, because of the ocular deviation, the visual message imprinted on the retina of the deviating eye is incompatible with that received by the fixing eye. Likewise, in anisometropia, the defocused image on the retina of the more ametropic eye cannot be fused with that originating from the fovea of the fixing eye. With unilateral deprivation amblyopia, only diffuse light can enter one eye and abnormal binocular interaction may occur between the sharply focused image originating in the non-deprived eye and the excitation resulting from diffuse light ("white noise") in the deprived eye. Obviously, with binocular visual deprivation, this mechanism cannot play a role, for the visual input to both eyes, albeit decreased, is similar. Thus, with all three types of amblyopia, dissimilarity of afferent input may lead to abnormal binocular interaction and subsequent blockage of transmission of visual excitation from the deviating, ametropic, or visually deprived eye to the visual cortex.

Recent findings from a study of experimental amblyopia in the rhesus monkey lend support to this concept. Amblyopia of varying degree was produced by unilateral lid suture or artificial esotropia in visually immature monkeys (von Noorden, Dowling, and Ferguson, I970a; von Noorden and Dowling, 1970). The response characteristics of neurons in the striate and pre-striate cortex of these monkeys were studied, and changes were observed at the single neuron level that parallelled the degree of behavioural changes. In an animal with severe amblyopia, only a small number of neurons were driven from the 
deprived or deviated eye. With less severe amblyopia, many neurons received input from that eye, but in all animals only a small number of neurons were binocular (Baker, Grigg, and von Noorden, in press). These data contrast sharply with those obtained from normal monkeys in which most striate and pre-striate neurons may be driven from either eye. This severe defect of binocular response characteristics of visual cortical neurons and the decreased neuronal response from the amblyopic eye in more severe forms of amblyopia were noted in monkeys with both strabismic amblyopia and stimulus deprivation amblyopia without strabismus, suggesting a common mechanism in both conditions. We also found a significant reduction of cell section areas in all layers of the lateral geniculate nucleus (LGN) that received input from the deprived or esotropic eye (von Noorden, 1973b). Of interest was the fact that the degree of cell shrinkage in the LGN correlated well with the number of cortical neurons that responded to stimulation from the deprived or esotropic eye (von Noorden and Middleditch, in preparation). Again, the histological changes in strabismic and stimulus deprivation amblyopia were essentially identical and, like the neurophysiological anomalies, differed only in the degree of severity.

Experiments in cats with unilateral lid suture have shown that geniculate cell growth may indeed be influenced by competitive interaction between normal and deprived cells; one cell group may affect the growth of another (Guillery and Stelzner, 1970; Guillery, I972). It is of interest that this cell shrinkage was shown to be reversible after enforced usage of the formerly deprived eye (Chow and Stewart, 1972). The implication of this finding with respect to the reversibility of certain forms of human amblyopia needs no further elaboration. In further support of the binocular competition theory of amblyopia is the finding of Wiesel and Hubel ( $1965 \mathrm{a}$ ) that cortical neurophysiological anomalies are less severe in kittens which are bilaterally deprived than in those with unilateral deprivation, and the observation of Guillery (1973) that bilateral lid suture in kittens may have relatively little effect on geniculate cell growth. We know from patients with bilateral congenital cataracts, in whom only stimulus deprivation but not abnormal binocular interaction is involved, that amblyopia is less severe than in patients with unilateral cataracts (von Noorden, Ryan, and Maumenee, 1970b).

At present we are studying these aspects further in monkeys, and on a preliminary basis have interpreted the histological anomalies in the LGN as being a perhaps reversible defect in the integrity of retinocortical connections caused by abnormal binocular interaction. Whether this defect is caused by interlaminar inhibition or is secondary to inhibition at a cortical level is not yet known, and both possibilities must be taken into account.

Time does not permit me to elaborate further on our findings in monkeys with experimental amblyopia. It suffices to say that, because of the anatomical and functional similarity of the visual system in monkeys and humans, one may reasonably assume that similar neurophysiological and structural anomalies occur in the human amblyopic patient

In summary, we propose that the primary factor in the production of amblyopia is a modification of normal visual experience during a period when the visual system is susceptible to such alterations. In spite of the aetiological difference in the nature of the underlying clinical conditions, common amblyopiogenic factors appear to be operative in strabismic, anisometropic, and form vision deprivation amblyopia. These causative mechanisms are stimulus deprivation by inadequate image formation on the fovea, abnormal binocular interaction by incongruity of the visual input received by the two eyes, and perhaps a combination of both factors. These conditions cause neurophysiological anomalies in the striate and prestriate cortex and morphological changes in the LGN of monkeys with experimental amblyopia, and we must assume that similar anomalies occur 
in the human visual system. Further study of neurophysiological and neuroanatomical characteristics of experimental amblyopia in an appropriate animal model, such as the monkey, is of fundamental importance in advancing our understanding of the site and mechanism of amblyopia in our patients.

\section{Discussion}

VAN SLUYTERS Although it is generally accepted that closure of the fellow eye by whatever means is a satisfactory therapeutic method of restoring development to an amblyopic eye, this has been shown to have devastating results from the binocular standpoint.

von NOORDEN Hubel and Wiesel (1963) showed several years ago that lid closure for only a matter of a few days in the visually immature kitten had a devastating effect on the integrity of cortical function. Stimulated by this observation, we performed this experiment in some of our monkeys from a behavioural point of view. One eye was closed for periods ranging from 2 to 4 weeks during visual immaturity, which ranges from o to 8 weeks in the monkey, not unlike that in the cat. The eye was then opened and the monkey allowed to grow up with both eyes open. The good eye was then closed and the acuity in the previously closed eye was found to be severely reduced, even down to light perception. One monkey had his eyelid closed after the period of susceptibility to visual deprivation and he recovered good vision when the eyelid was opened. Because of these results I am deeply concerned about the unilateral occlusion of visually immature infants for even brief periods. Examination of my clinical records revealed one child who had had one eye occluded as a baby for 4 weeks after a corneal abrasion; this eye was later found to have a visual acuity of only $20 / 60$, with no ocular abnormality. Another child had a marked purulent conjunctivitis from birth which caused complete lid closure of one eye for about 3 months. Shortly after the lids opened a marked esotropia developed, and in spite of patching for 6 months the visual acuity in that eye has not improved beyond 5/200. In view of these findings, occlusion should not be undertaken in an infant even for such short periods as a few days. This particularly applies to children under 6 months of age. If occlusion is necessary for any reason at all, then alternating occlusion of the opposite eye should be undertaken. This does not mean to say that if amblyopia is diagnosed it should not be treated vigorously. In the 2- to 3-yearold child the chance of developing deprivation amblyopia from short periods of occlusion is much less likely and occlusion therapy is eminently successful at that age.

PEARCE In view of these observations, should a child with complete congenital ptosis be operated on before the age of 6 months?

VON NOORDEN Yes. I have observed several cases of irreversible amblyopia with complete congenital ptosis especially if caused by a haemangioma. Fortunately, most patients with congenital ptosis receive some retinal stimulation by tipping the head backwards. The same considerations apply to uniocular congenital cataracts; the patients should be operated on and given a contact lens at the very earliest possible age.

PARKS I have been evaluating this problem for many years. My procedure is to operate on the cataract at the earliest possible moment, prescribe a contact lens for the aphakic eye within one month postoperatively, and occlude the unaffected eye. The best visual result so far is $20 / 40$, but without binocular vision. I doubt if any visual benefit has been obtained in patients treated by this method when they are over I year old.

WYBAR Uniocular congenital cataract must be operated on very early. Unless one does this, the fixation mechanisms never develop, and the chances of obtaining any reasonable visual acuity are therefore very slight. If you operate on any child more than 6 months old there is not the slightest hope of improving the visual acuity, however hard one tries by contact lens or by patching. There is no doubt that operating on congenital cataracts at a very young age is a very hazardous procedure, 
particularly if there had been previous rubella, and I do not feel that intraocular lenses are justified at this age, because of themselves they inevitably increase the hazards in an already difficult situation.

NUTT I feel it is rarely justified to operate on an uniocular congenital cataract at an early age largely because it is frequently associated with other intraocular abnormalities incompatible with a reasonable visual acuity.

VON NOORDEN There is no evidence that there is an increase in other congenital abnormalities in the eye in cases of a unilateral congenital cataract. It is much more likely that the failure to achieve a good visual result by surgery of congenital cataract is due to changes in the visual cortex. Although some cases will undoubtedly fail, others will do well if they are operated on within the first Io days of life. There is now some well-documented evidence of one or two patients who have done exceptionally well with this regimen, provided both parent and child are prepared to work hard to the final end.

BLAKEMORE During the period of plasticity there is a critical period at about 28 days in the kitten when the eyes are most sensitive to occlusion. Has Dr. von Noorden any evidence from his monkey experiments of a similar period in these animals, or of anything in man which corresponds to this particular fact?

VON NOORDEN A search of the literature failed to reveal any well-documented evidence. I therefore examined my own records and found in 9 years seven patients with evidence of deprivation amblyopia. The oldest patient observed developed deprivation amblyopia at the age of $5 \mathrm{I}$ months, which seems to be at the upper limit of the sensitive period in humans. In the monkey the sensitive period ranges from birth to 8 weeks.

ROMANES I have several cases of herpes simplex of the cornea in which amblyopia has developed because of corneal scarring. Is any treatment satisfactory?

VON NOORDEN I have reported similar cases in a paper with Maumenee (von Noorden and Maumenee, 1968). When these patients had keratoplasty at the age of 18 or 20 years the visual acuity did not improve even though they had a clear graft. I would not therefore hesitate to do a corneal graft in a very young child even accepting the risks that this might involve, because an eye with dense corneal scars is doomed unless one does something about it. I know of some patients who have done well. 